\title{
DESENVOLVIMENTO, COMPARAÇÃO E ANÁLISE DE MODELOS DE TRANSPORTE PNEUMÁTICO.
}

\author{
A. S. PEREIRA ${ }^{1}$, M. L. MAGALHÃES ${ }^{1}$, J. A. M. LEMOS $^{1}$ e S. J. M. CARTAXO ${ }^{1}$ \\ ${ }^{1}$ Universidade Federal do Ceará, Departamento de Engenharia Química \\ E-mail para contato: samuel@ufc.br
}

\begin{abstract}
RESUMO - O transporte pneumático utiliza correntes de gás para a movimentação de material particulado, o que lhe confere notada importância industrial, na medida em que muitas indústrias necessitam realizar a movimentação de cargas de material sólido. Diante disso, a elaboração de modelos matemáticos com vistas à simulação computacional se torna uma atividade que contribui para a redução de custos. O objetivo deste trabalho é realizar a modelagem matemática de um processo de transporte pneumático vertical para $\mathrm{o}$ estudo das variáveis operacionais de maior influência, comparativamente a modelos consagrados na literatura técnica. Foram realizadas simulações em software livre Python e seus módulos, de modo a avaliar a implementação de modelos matemáticos diversificados, tais como o uso da equação empírica de Ergun ou a equação de estado para gases ideais para resolver a pressão do sistema. A validação do modelo foi realizada de forma comparativa com estudos de casos reportados na literatura, e observou-se que o modelo que utiliza a equação de estado para predizer a variação de pressão se mostrou mais satisfatório e confiável. Os resultados mostram a influência das propriedades do fluido/partícula, os perfis de velocidades do fluido e das partículas e o perfil da queda de pressão.
\end{abstract}

\section{INTRODUÇÃO}

O arraste de material particulado através de tubulações, por meio de uma corrente gasosa, é uma operação unitária que ficou conhecida como transporte pneumático (Figura 1). Sua utilização é crescente e tornou-se comum desde a década de 20 (Klinzing et al., 1997).

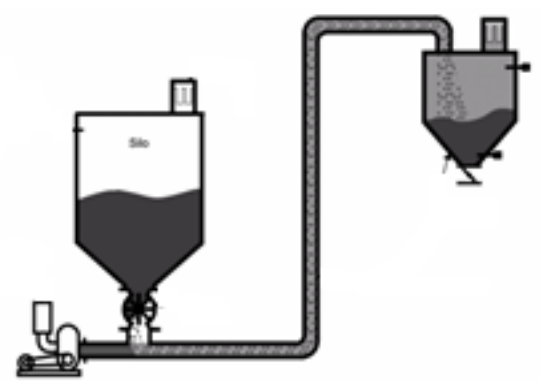

Figura 1 - Transporte pneumático. Fonte: Dynamic Air - Conveying Systems (2005). 
O transporte pneumático possui diversificada aplicação industrial, sendo algumas citadas por Marcus et al. (1990): craqueamento catalítico, combustão, produção de propileno, secagem, processamento de minério, indústrias alimentícias e farmacêuticas. Isso se deve à versatilidade no deslocamento de materiais sólidos, transporte limpo e isento de perdas, baixo custo de manutenção, fácil operação e excelente contato entre as fases gás-sólido.

Para projetar um sistema de transporte pneumático eficiente, faz-se necessário conhecer sua queda de pressão, a velocidade do gás de transporte e das partículas transportadas. Cabrejos et al. (1992) afirma que a velocidade mínima de transporte é um dos parâmetros mais importantes e que deve ser estimado para evitar elevados custos devido ao grande consumo de energia.

Neste trabalho serão apresentados modelos macroscópicos, para o transporte vertical, baseados na fluidodinâmica do sistema gás-sólido, utilizando fundamentos da mecânica dos fluidos e princípios termodinâmicos.

As suposições realizadas para o modelo unidimensional em regime permanente são: sistema isotérmico sem transferência de massa entre as fases; tamanho das partículas é uniforme possuem mesma massa especifica; partículas de mesmo diâmetro e densidades como uma fase contínua; e fase particulada incompressível. Sob estas hipóteses, foi desenvolvida uma versão modificada do modelo proposto por Gidaspow (1994), conforme abaixo.

\subsection{Equações da Continuidade}

\section{Fase gasosa:}

$\frac{\partial\left(\rho_{g} \cdot \varepsilon \cdot v_{g}\right)}{\partial z}=0$

$\left(\varepsilon \cdot \rho_{g} \cdot \frac{\partial\left(v_{g}\right)}{\partial z}+v_{g} \cdot \rho_{g} \cdot \frac{\partial(\varepsilon)}{\partial z}+\varepsilon \cdot v_{g} \cdot \frac{\partial\left(\rho_{g}\right)}{\partial z}\right)=0$

Fase particulada:

$\frac{\partial\left(\rho_{p} \cdot(1-\varepsilon) \cdot v_{p}\right)}{\partial z}=0$

$\rho_{p} \cdot\left((1-\varepsilon) \cdot \frac{\partial\left(v_{p}\right)}{\partial z}-v_{p} \cdot \frac{\partial(\varepsilon)}{\partial z}\right)=0$

\subsection{Equações do Momentum}

Fase gasosa:

$\frac{\partial\left(\rho_{g} \cdot \varepsilon \cdot v_{g}{ }^{2}\right)}{\partial z}=-\frac{\partial(\varepsilon \cdot P)}{\partial z}-F_{D z}-F_{G g}$ 
2. $\rho_{g} \cdot \varepsilon \cdot \frac{\partial\left(v_{g}\right)}{\partial z}+\rho_{g} \cdot v_{g}{ }^{2} \cdot \frac{\partial(\varepsilon)}{\partial z}+\varepsilon \cdot v_{g}{ }^{2} \cdot \frac{\partial\left(\rho_{g}\right)}{\partial z}+\varepsilon \cdot \frac{\partial P}{\partial z}+P \cdot \frac{\partial \varepsilon}{\partial z}+F_{D z}+F_{G g}=0$

Fase particulada:

$\frac{\partial\left(\rho_{p} \cdot(1-\varepsilon) \cdot v_{p}^{2}\right)}{\partial z}=-\frac{\partial((1-\varepsilon) \cdot P)}{\partial z}+F_{D z}-F_{G p}$

2. $\rho_{p} \cdot(1-\varepsilon) \cdot \frac{\partial\left(v_{p}\right)}{\partial z}-\rho_{p} \cdot v_{p}{ }^{2} \cdot \frac{\partial(\varepsilon)}{\partial z}+(1-\varepsilon) \cdot \frac{\partial P}{\partial z}-P \cdot \frac{\partial \varepsilon}{\partial z}-F_{D z}+F_{G g}=0$

\subsection{Equações Constitutivas}

\section{Fase gasosa:}

Equação de estado

$$
\begin{aligned}
& \rho_{g}=\frac{M M}{R \cdot T} \cdot P \\
& \frac{\partial P}{\partial z}=\frac{R \cdot T}{M M} \cdot \frac{\partial \rho_{g}}{\partial z}
\end{aligned}
$$

Equação de Ergun (1952)

$\frac{\partial P}{\partial z}=\frac{1.75 \rho_{g}}{d_{p}}(1-\varepsilon)\left(v_{g}-v_{p}\right)^{2}+\frac{150 \mu_{g}}{\varepsilon \cdot d_{p}{ }^{2}}(1-\varepsilon)^{2}\left(v_{g}-v_{p}\right)$

Fase particulada:

$\rho_{p}=\rho_{p 0}=$ const

\subsection{Forças}

Para este modelo, foram consideradas a força gravitacional e a força de arraste. Gidaspow (1994) propôs que a força gravitacional age de forma proporcional a massa da partícula e que a força de arraste é correlacionada dependendo da porosidade do sistema. Para a fase densa, a força de arraste pode ser obtida pela Equação de Ergun (1952) e para a fase dispersa pela equação de Richardson e Zaki (1954), que estima o coeficiente de arraste gás-partícula como função do número de Reynolds (Rowe e Henwood, 1961).

\section{Força de arraste:}

$$
\begin{array}{ll}
F_{D z}=\frac{1.75 \rho_{g}}{d_{p}}(1-\varepsilon)\left(v_{g}-v_{p}\right)^{2}+\frac{150 \mu_{g}}{\varepsilon \cdot d_{p}{ }^{2}}(1-\varepsilon)^{2}\left(v_{g}-v_{p}\right) & 0.2 \leq \varepsilon<0.8 \\
F_{D z}=\frac{3}{4} \frac{C_{d z} \cdot(1-\varepsilon) \varepsilon^{-2,67} \cdot\left(v_{g}-v_{p}\right) \cdot\left|v_{g}-v_{p}\right| \cdot \rho_{g}}{d_{p}} & 0.8 \leq \varepsilon
\end{array}
$$


Fase gasosa:

$F_{G g}=\varepsilon \cdot \rho_{g} \cdot g$

$\underline{\text { Fase particulada: }}$

$F_{G p}=(1-\varepsilon) \cdot \rho_{p} \cdot g$

\section{COMPARAÇÃO E ANÁLISE DE MODELOS DE TRANSPORTE PNEUMÁTICO}

Observa-se que o modelo proposto possui cinco equações diferenciais e cinco variáveis, sendo elas: porosidade, densidade do gás, velocidade do gás, velocidade da partícula e pressão. Para resolver o sistema de equações foi utilizado framework numérico Python e seus módulos interfaceados através do IPython Notebook.

Em caráter comparativo dos modelos diferenciais desenvolvidos neste trabalho, foi selecionado como benchmark o estudo de caso experimental de Zenz (1949), referenciado por Arastoopour e Gidaspow (1979). Todos os parâmetros relativos ao sistema de transporte pneumático estão apresentados na Tabela 1, assim como as condições inicias na Tabela 2.

Tabela 1 - Dados dos experimentos.

\begin{tabular}{c|c|c|c|c|c|c}
$\rho_{p}\left(\mathrm{Kg} / \mathrm{m}^{3}\right)$ & $\mu_{g}$ (Pa.s) & D.do tubo $(\mathrm{m})$ & $L(\mathrm{~m})$ & $d_{p}(\mathrm{~m})$ & $M M(\mathrm{Kg} / \mathrm{mol})$ & $T(\mathrm{~K})$ \\
\hline $1.84 .10^{-5}$ & $1.6710^{-3}$ & $4.4510^{-2}$ & 1.20 & $1.6710^{-3}$ & 0.029 & 303.15
\end{tabular}

Tabela 2 - Condições iniciais dos experimentos.

\begin{tabular}{c|c|c|c|c}
$\rho_{g}\left(\mathrm{Kg} / \mathrm{m}^{3}\right)$ & $v_{g}(\mathrm{~m} / \mathrm{s})$ & $v_{p}(\mathrm{~m} / \mathrm{s})$ & $\varepsilon$ & $P(\mathrm{~Pa})$ \\
\hline 3.03 & 25.0 & 0.56 & 0.9 & $26.410^{4}$
\end{tabular}

A Figura 2 ilustra o comportamento axial dos experimentos de Zenz (1949) para o modelo desenvolvido por Arastoopour e Gidaspow (1979). As simulações mostradas na Figura 3 apresentam notáveis diferenças no comportamento dos perfis de velocidades gás/partícula, onde: (a) desconsiderase a variação de pressão e utiliza-se a correlação empírica de Ergun, comumente empregada para descrever a variação de pressão por unidade de comprimento, na força de arraste e (b) princípios termodinâmicos para predizer a variação da pressão no sistema; (c) apresenta o modelo desenvolvido por Adewnmi e Arastoopour (1986), que considera o gradiente de pressão apenas na fase gasosa.

Observa-se que o modelo que emprega a equação de Ergun não se mostrou adequado para o sistemas de transporte pneumático, indo de encontro a física do processo. Os modelos que utilizam a equação de estado, quando comparado a Figura 2, mostraram-se mais satisfatórios, sendo o modelo diferencial desenvolvido neste trabalho o que apresentou mesmo comportamento nos perfis de velocidades e pequenos desvios nas velocidades finais. 


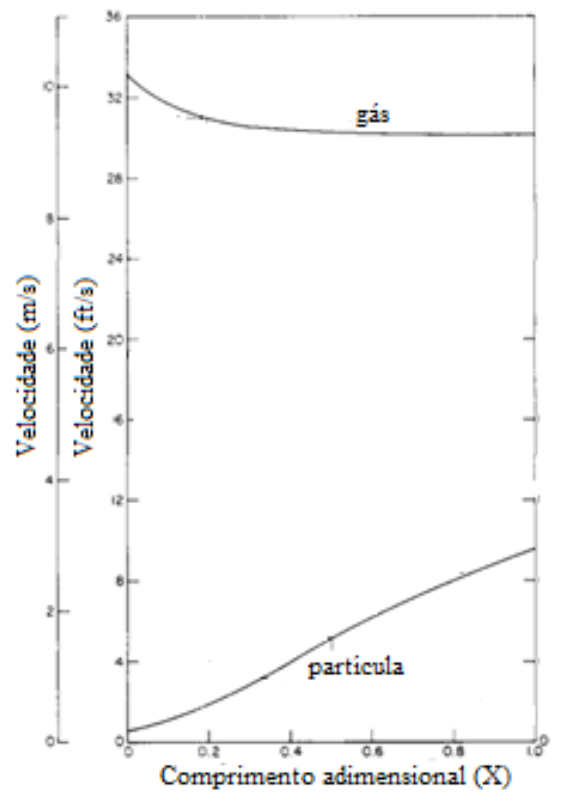

(a)

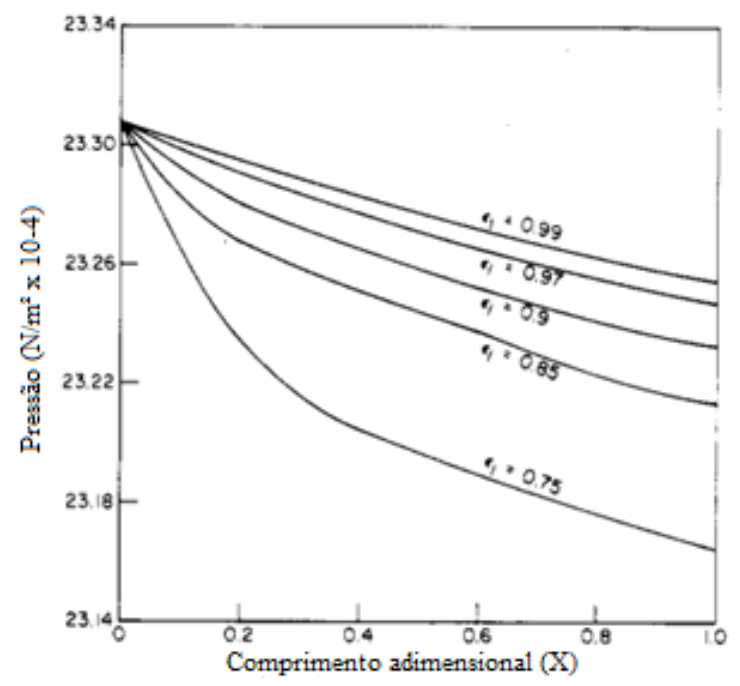

(b)

Figura 2 - Modelo desenvolvido por Arastoopour e Gidaspow (1978) que prevê o comportamento axial dos experimentos de Zenz (1949); (a) Perfis de velocidades e (b) Perfil de Pressão.

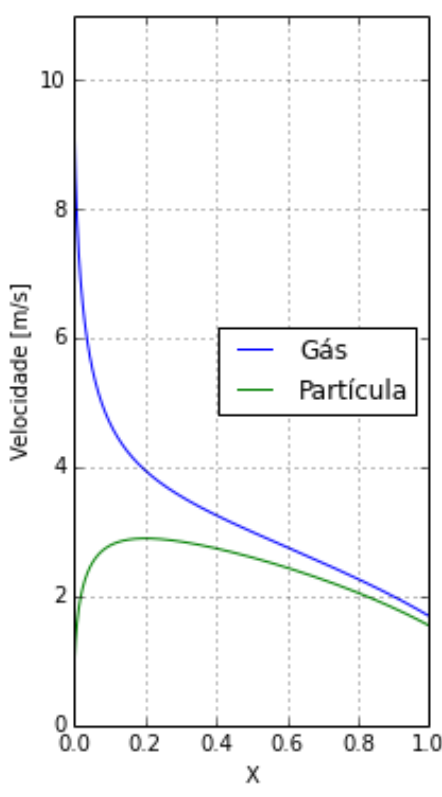

(a)

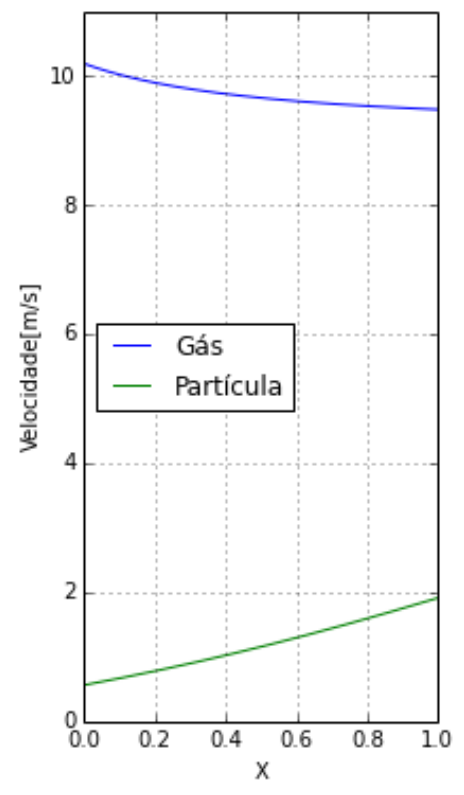

(b)

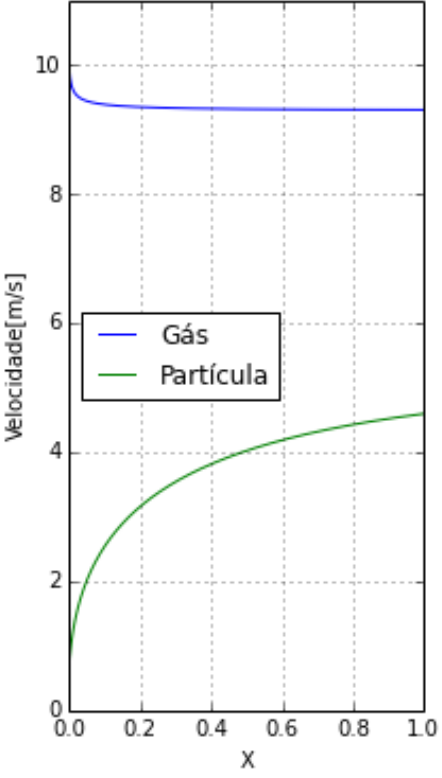

(c)

Figura 3 - Perfis de velocidades (a) Equação de Ergun; (b) Equação de estado e (c) Modelo de Adewnmi e Arastoopour (1986), considerando somente a variação axial. 
A seguir, são mostrados na Figura 4, os perfis da porosidade, densidade e pressão para o modelo dinâmico validado. No sistema experimental, a pressão registrada foi constante, e consequentemente não se determinou o efeito da densidade do gás. O resultado obtido confirma que a variação da pressão para esse sistema é muito pequena, mas podem-se perceber os efeitos da densidade no gás no perfil da queda de pressão.

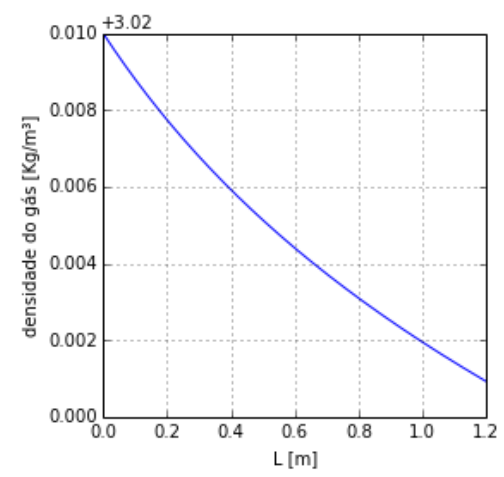

(a)

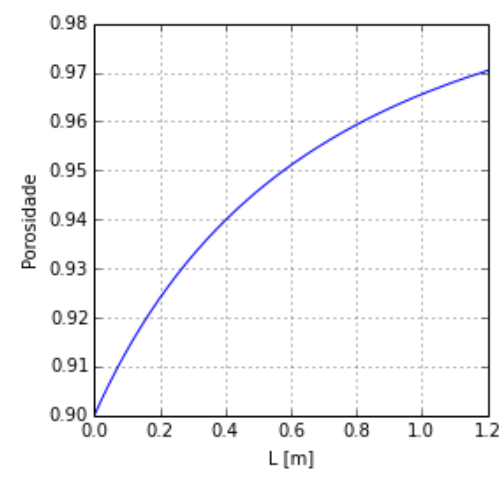

(b)

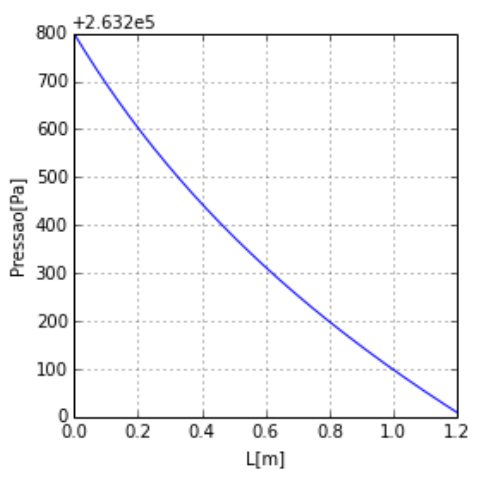

(c)

Figura 4 - Perfil da (a) Desidade do gás; (b) Porosidade e (c) Pressão.

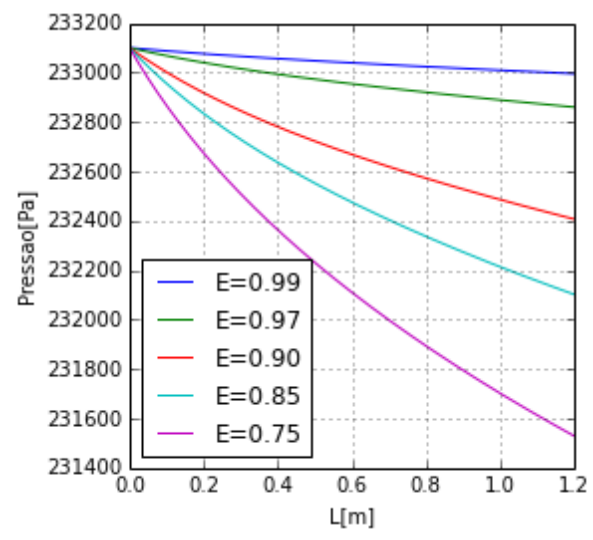

Figura 5 - Comportamento da pressão com variação da porosidade.

A Figura 5 apresenta o comportamento da pressão com a variação da porosidade, que condiz com os experimentos de Zenz (1949) e pode ser explicado através da força de arraste. Quando é feito o transporte em fase densa (porosidade $<0.8$ ), a força de arraste é maior, quando comparada a fase dispersa, ocasionando uma maior perda de pressão. Conclui-se que quanto menor a porosidade, maior a variação de pressão para o mesmo comprimento.

Outro estudo de caso foi realizado comparando o modelo desenvolvido por Theologos e Markatos (1994) para os dados experimentais de Hariu e Molstad (1949). Verifica-se pela Figura 6, que o modelo diferencial estudado não apresenta desvios exacerbados, podendo ser consequência do nivelamento pela média radial. Experimentalmente, o valor obtido para a pressão depois de $1.026 \mathrm{~m}$ foi de $149 \mathrm{~Pa}$, mostrando a influência da variação das propriedades ao longo do raio. 


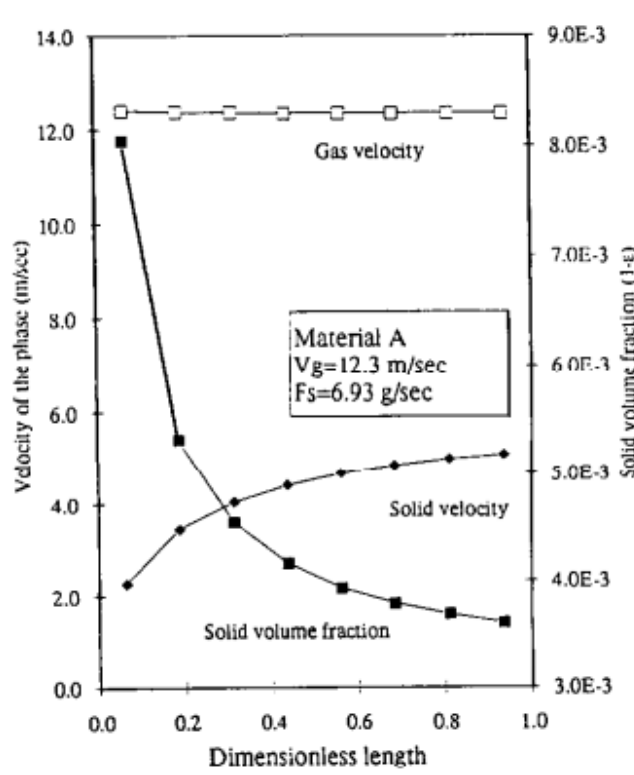

(a)

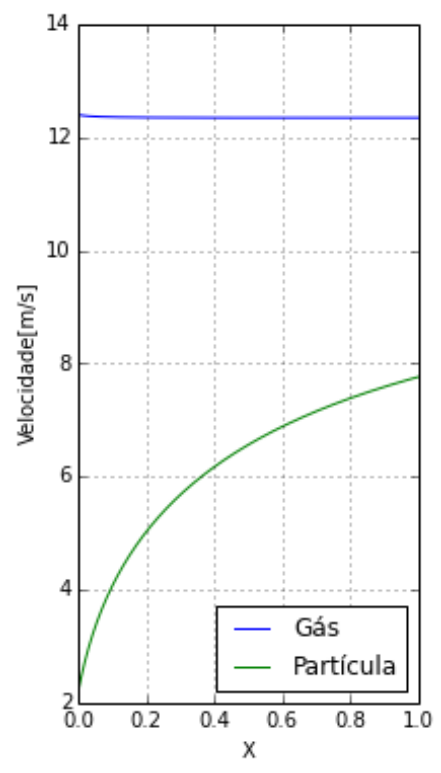

(b)

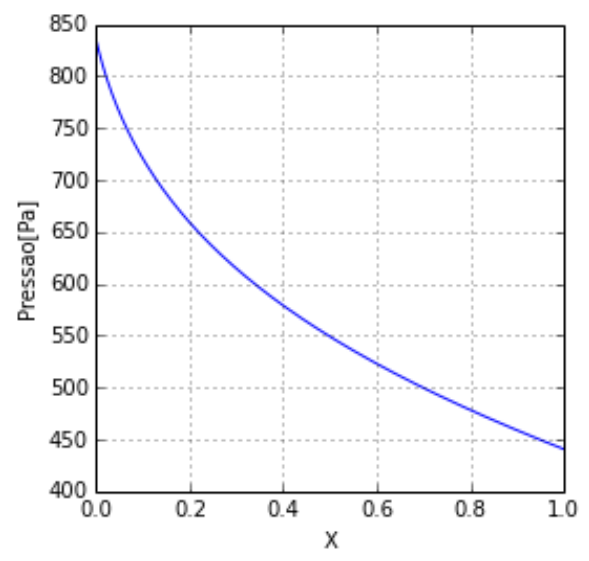

(c)

Figura 6 - (a) Perfil de velocidade para os experimentos de Hariu e Molstad (1949); (b) Perfil de velocidade para o modelo desenvolvido neste trabalho; (c) Perfil de pressão.

\section{CONCLUSÃO}

O presente trabalho explorou possibilidades de modificação do modelo matemático de transporte pneumático proposto por Gidaspow (1994). Os resultados foram comparados com dados experimentais reputados como confiáveis de Zenz (1949) e de Hariu e Molstad (1949). A representação da queda de pressão ao longo do tudo foi realizada por meio de equação de estado e da equação de perda de carga de Ergun. Os perfis de velocidade obtidos usando-se a equação de Ergun não se mostraram fisicamente realistas. A validação do modelo matemático foi satisfatória e compatível com os resultados fornecidos pela modelagem de Gidaspow (1994).

\section{NOMENCLATURA}

$C_{d z}=$ coeficiente de arraste axial

$d_{p}=$ diâmetro da partícula $(\mathrm{m})$

$F_{D z}=$ força de arraste na direção axial $(\mathrm{Pa} / \mathrm{m})$

$F_{G g}=$ força gravitacional para o gás $(\mathrm{Pa} / \mathrm{m})$

$F_{G p}=$ força gravitacional para a partícula $(\mathrm{Pa} / \mathrm{m})$

$v_{g}=$ velocidade do gás $(\mathrm{m} / \mathrm{s})$ 
$v_{p}=$ velocidade da partícula $(\mathrm{m} / \mathrm{s})$

$\varepsilon=$ porosidade

$\rho_{g}=$ densidade do gás $\left(\mathrm{kg} / \mathrm{m}^{3}\right)$

$\rho_{p}=$ densidade da partícula $\left(\mathrm{kg} / \mathrm{m}^{3}\right)$

$\mu_{g}=$ viscosidade do gás ( $\mathrm{Pa} \mathrm{s}$ )

\section{REFERÊNCIAS}

ADEWUMI, M. A.; ARASTOOPOUR, H. Two-Dimensional Steady State Hydrodynamic Analysis of Gas-Solids Flow in Vertical Pneumatic Conveying Systems, J. Chem. Phys., v. 31, p. 459-466, 1986.

ARASTOOPOUR, H.; GIDASPOW, D. Vertical Pneumatic Conveying Using Four Hydrodynamic Models. Ind. Eng. Chem. Fundam., v. 18, p. 123-130, 1979.

CABREJOS, F. J. and KLINZING, G. E. Incipient motion of solid particles in horizontal Pneumatic conveying. Powder Technology. v. 72, p.51-61, 1992.

DYNAMIC AIR LTDA. Conveying Systems. Nazaré Paulista, Princípios fundamentais do transporte pneumático, São Paulo: Bulletin 9515-4-BR, 2005.

ERGUN, S. Fluid flow through packed columns, Chemical Engineering Progress, v.48, p.89-94, 1952.

GIDASPOW, D. Multiphase flow and fluidization: continuum and kinetic theory descriptions, Boston: Academic Press Inc., 1994.

HARIU, O. H.; MOLSTAD, M. C. Pressure drop in vertical tubes vertical tubes in transport of solids by gases. Ind. Eng. Chem., v. 41, p. 1148-1160, 1949.

KLINZING, G. E.; MARCUS, R. D.; RIZK, F.; LEUNG, L. S. Pneumatic conveying of solids: $a$ theoretical and practical approach. London: Chapman \& Hall, 1997.

MARCUS R. D., LEUNG L. S., KLINZING G. E., RIZK F. Pneumatic conveying of solids. a theoretical and practical approach. London: Chapman and Hall, 1990.

RICHARDSON, J. F.; ZAKI, W. N. Sedimentation and Fluidization: Part I. Trans. Instn. Chem. Engrs., v. 32, n' 35, 1954.

ROWE, P.N. Drag forces in a hydraulic model of a fluidised bed - part II, Trans. Instn. Chem. Engrs, v.39, p.175-180, 1961.

THEOLOGOS, K. N.; MARKATOS, N. C. Modelling of vertical pneumatic-conveying hydrodynamics. Appl. Math. Modelling, v. 18, p. 306-320, 1994.

ZENZ, F. A.; Two-phase fluid-solid flow. The Canadian Journal of Chemical Engineering, v. 41, p. 20801-2806, 1949. 